\title{
Carnets
}

Revue électronique d'études françaises de l'APEF

Deuxième série - $10 \mid 2017$

Pratiques de l'errance, vécus de la mémoire

\section{Autour de quelques concepts et de leurs prolongements dans la littérature judéo- maghrébine}

Bonheurs de l'errance / trauma de l'exil

\section{Guy Dugas}

\section{OpenEdition}

\section{Journals}

Édition électronique

URL : http://journals.openedition.org/carnets/2178

DOI : $10.4000 /$ carnets. 2178

ISSN : 1646-7698

Éditeur

APEF

Référence électronique

Guy Dugas, « Autour de quelques concepts et de leurs prolongements dans la littérature judéo-

maghrébine ", Carnets [En ligne], Deuxième série - 10 | 2017, mis en ligne le 30 avril 2017, consulté le 19 avril 2019. URL : http://journals.openedition.org/carnets/2178; DOI : 10.4000/carnets.2178

Ce document a été généré automatiquement le 19 avril 2019.

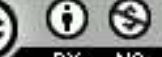

Carnets est mis à disposition selon les termes de la licence Creative Commons - Atribution - Pas d'utilisation commerciale 4.0 International. 


\section{Autour de quelques concepts et de leurs prolongements dans la littérature judéo-maghrébine}

Bonheurs de l'errance / trauma de l'exil

\section{Guy Dugas}

1 J'ai toujours considéré qu'avant d'analyser ou d'étudier un phénomène, avant d'approfondir une question ou même d'aborder une œuvre, il y avait lieu de dresser des bibliographies, de revenir sur quelques définitions, sur quelques concepts essentiels à cette approche, à cette étude. Nécessité herméneutique qui me paraît d'autant plus grande lorsque le domaine abordé est en pleine évolution conceptuelle et en totale remise en cause, comme cela me semble être actuellement le cas des littératures francophones avec l'apparition de nouvelles notions (« littérature-monde ", « littérature migrante ", "littératures exilique, post-exilique », etc.) et de nouvelles théories venant renouveler nos approches (" postcolonial studies », « subaltern studies ", etc).

De même que, dès 1992, j'avais proposé un « Retour sur quelques concepts mal établis " ${ }^{1}$, dans le contexte de ce que nous nommions alors les «littératures francophones »- de même en ouverture de ce volume d'actes, j'ai l'intention de proposer une remise en question de trois ou quatre notions-clés ; j'en rajouterai même une ou deux, inspirées par la lecture du programme et de certaines présentations de communications du colloque de Bragance.

3 Pour cela, j'aurai bien soin de prendre systématiquement appui sur des textes littéraires, pour la plupart empruntés au corpus maghrébin ou judéo-maghrébin - on ne se refait pas et vous savez que c'est là le domaine sur lequel je travaille depuis près de 40 ans - mais pas que... 


\section{Errance vs nomadisme}

4 Depuis L'Iliade, Don Quichotte et les romans de chevalerie du Moyen-Âge, le thème de l'errance est constant en littérature, que ce soit dans le roman ou dans la poésie - plus particulièrement dans la poésie africaine en général, pour peu qu'elle remonte aux sources orales qui l'ont produite.

5 À en croire la plupart des dictionnaires, « errance » et « nomadisme » devraient être considérés comme synonymes : «Absence, pour une personne ou un peuple, de résidence ou de destinations fixes » - voilà ce qu'ils nous disent, associant l'individuel au collectif et chargeant ces deux contextes d'émotions et d'a-normalité, ne serait-ce qu'à travers le terme d'absence. Il est vrai qu'à partir de sa cinquième édition (1798), le Dictionnaire de l'Académie prend soin de préciser que « nomade » « ne se dit que des nations », avant de relativiser dès les éditions suivantes : "se dit surtout des nations, des tribus, des peuplades ".

\subsection{A propos de l'errance}

6 On peut donc poser que l'errance serait d'ordre individuel et relèverait d'une condition Don Quichotte et le picaro sont des personnages errants - tandis que le nomadisme renverrait plutôt à un phénomène sociologique ou communautaire : telle ou telle tribu ou peuplade est dite nomade...

7 Mais ces communautés ou personnes "sans résidence ou destination fixes ", que nous les appelions « errants » ou " nomades », sont parfaitement libres dans leur errance; elles peuvent emporter avec elle leurs valeurs culturelles ou religieuses, leur langue, leurs traditions, et parfaitement s'en satisfaire...

8 Au plan personnel, l'errance n'est porteuse a priori d'aucun état d'âme particulier. Si pour les uns, certes, elle semble née d'une condamnation, voire d'une malédiction historique - on connait le mythe du Juif errant - pour beaucoup d'autres elle demeure synonyme de découverte et de libération de tous les liens, voire de toutes les convenances : ainsi le Clézio, Montherlant aussi durant toute une période de sa vie, se revendiquent-ils libres et heureux dans une errance librement choisie, éprouvant - selon l'expression d'André Gide - « la joie qu'il y aurait à ne plus se sentir d'attaches, de racines, si vous voulez » (Gide, 1898). Liberté de comportement qui à son tour détermine une liberté de l'écriture : c'est bien durant leurs périodes d'errance que Montherlant et Gide ont conçu les œuvres les plus libres (Amyntas, Moustique) ou les plus engagées (Voyage au Congo suivi de Le Retour du Tchad, La Rose de sable).

\subsection{A propos du nomadisme}

9 Idem pour le nomadisme : seuls notre mode de vie moderne, la constitution d'étatsnations, la fixation de frontières linguistiques et géographiques ont rendu ce fonctionnement social anachronique. Dès lors, ce sont les états qui n'ont eu de cesse de sédentariser les nomades, considérés comme un danger pour l'unité nationale, de les soumettre de plus ou moins grande force à leur culture et à leurs lois - comme le montre bien l'écrivain algérien Mouloud Mammeri dans un roman trop méconnu: La Traversée (Mammeri, 1989). 
10 Je voudrais introduire ici une rapide parenthèse : il est des théories selon lesquelles le nomadisme constituerait l'état naturel de l'homme, la condition première de toute société. La sédentarisation ne correspondrait qu'à une période relativement limitée, et pas la plus constructive ni la plus heureuse, de la longue histoire de l'humanité. Telle est, largement reprise de Michel Maffesoli (1997),la thèse de Jacques Attali (2003) dans un ouvrage récent, L'Homme nomade, dans lequel il démontre que c'est dans l'état de nomadisme que l'homme s'est toujours montré le plus créatif, le plus ouvert à l'autre, que se sont développés les arts, la musique, les sciences humaines ; c'est au contraire dans la sédentarité, l'affirmation de ses racines identitaires que l'homme a tendance à nier les différences, à mépriser d'autres cultures ou religions que la sienne, à faire preuve d'hétérophobie; c'est la sédentarité qui a permis la création de concepts lourds de conséquence : "racines ", «identité ", "patrie ", «langue maternelle ", etc. Que l'on songe seulement à la formation complexe du terme "patrie ", à la fois "père " par l'étymologie et « mère " par le genre, et à tout ce à quoi cela renvoie de fort et attachant.

11 L'écrivain tuniso-canadien de langue française Hèdi Bouraoui parle de nomaditude, état d'esprit conduisant le nomade à accueillir comme autant d'enrichissements les multiples identités que ses déplacements proposent, de les adopter sans les rejeter, car cela ne le dépossède pas de son identité native : «Ce processus fait sa richesse, surtout quand les différentes identités sont assumées dans la sérénité et non dans le déchirement, ni dans la désintégration de la mémoire. »(Bouraoui, 1994 : 9).

\section{Exil et Diasporas}

Retour à la définition des dictionnaires :

- Exil : situation faite à une personne ou à un groupe de devoir rompre avec leur société d'origine, d'eux-mêmes ou par suite d'un bannissement, d'une condamnation. Ne pas oublier : si errance et nomadisme représentent deux états ou comportements, individuels ou collectifs, l'exil au sens strict trouve d'abord sa définition dans le droit, la juridiction romaine distinguant exil, c'est-à-dire expulsion pure et simple d'une personne de son lieu de résidence ; relégation ou confinement ( = assignation à une résidence, le plus souvent aux marges (aux confins du territoire) et la déportation. Bien sûr, on peut aussi, comme Victor Hugo ou Stephan Zweig, choisir l'exil sans y avoir été le moins du monde condamné.

Mais l'exil ne suppose pas obligatoirement l'exode et l'on peut se sentir en exil dans son propre pays. Je prendrai ici en exemple le droit colonial français (largement inspiré du droit romain, du reste) lorsqu'il vient à s'opposer à certains cas de rébellion dans ses colonies : par exemple Abd el Kader en 1847 ou la révolte dite des Mokrani en 1871. Dans les deux cas, les révoltés (pas Mokrani puisqu'il meurt au combat, mais ceux qui l'entourent, notamment le Cheikh el Haddad et sa famille) sont parfois condamnés à la relégation (Abd-el-Kader) parfois à la déportation (Aziz el Haddad) mais ce n'est pas tout : leur famille restant au pays est aussi condamnée : d'une part à perdre ses terres, qui sont redistribuées à des colons ; d'autre part - c'est notamment le cas des enfants du Cheikh el Haddad, à la suite de sa déportation en Nouvelle-Calédonie - à voir ses enfants scolarisés éloignés de leur milieu natal pour être inscrits de force au Lycée National d'Alger où ils suivront bien sûr un enseignement en français. Pendant que le père est confiné ou déporté, les enfants sont donc, à proprement parler, exilés dans leur propre pays, exilés dans la langue de l'autre. 

donc rupture, arrachement: au milieu natal, à une langue maternelle, au contexte dans lequel on a grandi... arrachement et assignation : à résidence, à la pratique d'une langue, à un profond changement culturel, etc.

(ne l'exil est toujours ressenti comme une forme de violence (ne dit-on pas d'une personne qu'elle est frappée d'exil ?) et qu'il suscite la nostalgie d'un état et d'un temps antérieurs (le fameux "nostos " d'Homère dont je reparlerai lorsque j'aborderai la question de l'écriture de la mémoire).

Diaspora: Autre terme à significations multiples et évolutives, particulièrement ces derniers temps au gré des "transnational" et "cultural studies" "(on parle même quelquefois de "diaspora studies" !) Pour faire vite et simple, c'est à l'ouvrage-somme de Dufoix (2011) : La Dispersion, que j'emprunterai ici, pour ne retenir parmi ce que l'auteur nomme "les conditions de possibilité » des usages du terme que ceux intéressant mon propos - en particulier la diaspora juive avec les notions de "kappara" et de "galouth", sur lesquelles je reviendrai. Retenons également la relation ambivalente entre les deux termes, car s'il n'y a pas de diaspora sans exil, on peut très bien - on vient de le voir - se sentir exilé sans être extradé, placé en situation diasporique.

situation de communauté en diaspora pose en outre le problème de l'usage d'une langue et de son maintien face aux langues hégémoniques du pays d'accueil : cas du yiddish pour les diasporas juives en Europe ou du passage de l'italien au français chez les écrivains de la minorité italienne dans la Tunisie sous Protectorat ${ }^{2}$.

\subsection{Errance et exil}

19 Parce qu'il n'est jamais voulu par celui qui le vit, ou alors décidé à contrecœur, l'exil, qu'il soit individuel ou collectif, représente une situation toujours traumatisante. On connaît la définition d'Edward Saïd : «L'exil est la fissure à jamais creusée entre l'être humain et sa terre natale, entre l'individu et son vrai foyer, et la tristesse qu'il implique n'est pas surmontable. » (Saïd, 2008 : 241)

20 Certes, il est des individus ou des communautés ethniques ou confessionnelles qui choisissent de quitter le foyer qu'ils ont contribué à fonder, ou leur patrie natale. Afin de se désolidariser d'une politique, de s'opposer à des pratiques heurtant leurs convictions religieuses ou morales, de fuir une guerre... bref, de «s'éveiller du cauchemar de l'Histoire ", selon l'expression de Stephen Dédalus dans l'Ulysses de Joyce. Mais, si cela peut être consenti, ça ne se fait jamais de gaîté de cœur. Il peut y avoir - nous l'avons noté - du bonheur, voire de la passion dans le nomadisme et l'errance, qui sont l'un et l'autre fondés sur un désir d'ailleurs; il n'y a que regret et sentiment de malheur dans l'exil, qui est " empêchement d'être là où l'on désire être " (Bourque, Hogikyan, 2010: 2) - ce « là " pouvant être un lieu géographique, un espace vital, mais aussi une communauté ethnique, religieuse, linguistique, intellectuelle, etc.

... ce qui ne signifie pas, loin de là, qu'il s'agit d'une situation anesthésiante, que l'exilé vit constamment dans la négation et le rejet. L'exil, on le sait peut-être, au même titre que l'errance quoique dans un tout autre registre, une source de création très féconde.

Carnets, Deuxième série - $10 \mid 2017$ 


\subsection{Rapport à la patrie et à la langue}

Parce que ces situations d'exil et d'errance apparaissent fréquemment au sein de l'étude des expressions francophones, je voudrais revenir à présent sur les rapports que ces expressions entretiennent avec la patrie et la langue d'une part, avec le temps d'autre part - deux rapports souvent associés par la critique, quoique déterminés différemment, selon moi, dans l'une et l'autre situations :

- Revenons sur la nouvelle catégorisation « littératures migrantes » ou « littératures de la migration ", très en vogue aujourd'hui mais dont je ne sais guère ce qu'elle recouvre exactement, et qui du reste me semble parfois contredire le concept de «littératuremonde » cher à Michel Le Bris et Jean Rouaud. S'agit-il d'une nouvelle appellation des littératures des immigrations? Des écritures nomades célébrées par ce même Michel Le Bris au cours de son festival Étonnants voyageurs? Ou bien des littératures d'exil (je pense là à certaines entrées du Dictionnaire des écritures migrantes d'Ursula Mathis et Birgit Baumgarten (2012) - lequel exil pourrait précisément être considéré comme l'antonyme d'une situation de migration puisqu'il contraint l'écrivain à occuper dans un immobilisme relatif (Memmi ne se définit-il pas dans l'un de ses derniers essais comme un «nomade immobile » ?) un espace rejeté, depuis lequel il s'exprime selon une inspiration rétrovertie vers d'autres espaces, d'autres temps infiniment regrettés ${ }^{3}$ ? L'errant, le nomade n'ont pas de patrie - c'est ce qui fait leur liberté. Déchiré, l'exilé balance sans cesse entre une patrie perdue mais jamais oubliée et une patrie d'adoption qui ne l'accepte pas (ou/et que lui n'accepte pas). Au bout du compte, il se fabrique ce que ce

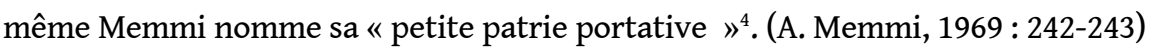

Parlons à présent de la rupture de l'intellectuel avec sa langue maternelle, de cet « exil dans la langue " que l'on évoque tant à propos des expressions francophones. Cette rupture peut, semble-t-il, se faire de deux manières :

Soit le devenir historique de la nation fait de tel ou tel écrivain en contexte postcolonial un «bâtard culturel » (l'expression est de Jean Amrouche, mais tant d'autres intellectuels maghrébins l'ont reprise, dans les années qui ont suivi les indépendances nationales) parce que sa propre histoire ou celle de sa famille ne lui ont pas permis d'être en harmonie avec ce que les états émergents se sont empressés, lorsque par chance ils l'ont $\mathrm{pu}$ (ce qui ne fut pas le cas dans tous les pays, en Afrique Noire notamment), d'appeler l'idiome majoritaire « langue nationale ». Et - même revenu au milieu de son peuple - il en conçoit un sentiment d'isolement, d'exil dans la langue.

Soit, dans d'autres contextes, cette rupture de l'intellectuel acculturé avec sa langue maternelle peut être considérée de façon totalement différente. Prenons le cas de Nancy Huston, écrivaine canadienne de langue maternelle anglaise qui a grandi aux États-Unis avant de s'installer en France. Voici ce qu'elle dit de son évolution entre sa langue maternelle et sa langue d'adoption :

C'est en français, à Paris, que j'ai osé mes premiers pas dans l'écriture : j'éprouvais un sentiment d'impunité ; mes parents ne liraient pas mes livres. Mais je prenais plus de risques car, hors de sa langue maternelle, on ne sait jamais quand on est au bord du cliché (...). En 1986, un travail sur l'exil a libéré en moi la nostalgie. En ne parlant, en ne chantant jamais en anglais à ma fille, j'ai compris que je perdais une partie de mon enfance (...). Ma maturité d'écrivain dans la fiction va de pair avec ces retrouvailles avec la langue maternelle. (Huston, 1997 : 43) 
Double liberté, donc: liberté d'une écriture transgressive grâce à la mise à distance que permet le nomadisme linguistique ${ }^{5} .$. mais aussi liberté d'une écriture régressive (" régression temporelle ", au sens freudien du terme) à laquelle aspirent les écrivains qui peuvent écrire dans deux langues, comme Tahar Ben Jelloun ou Rachid Boudjedra ; ou que regrettent ceux qui, comme Leïla Sebbar (2003), « ne parle[nt] pas la langue de [leur] père ", tout en reconnaissant que c'est précisément cela qui les a constitués en tant qu'écrivains ${ }^{6}$.

\subsection{Rapport au temps}

Autre distinction à opérer : le temps ne peut être vécu de la même manière en situation d'errance ou d'exil : porté vers l'autre ou vers un but à atteindre, le nomade se projette sans cesse vers l'avant, tournant le dos à son passé et ignorant superbement l'Histoire et ses leçons. Dans les romans de l'errance que sont Mangeclous et Les Valeureux d'Albert Cohen ou encore Le Désert d'Albert Memmi que nous analyserons rapidement plus loin, les personnages, tels des picaros des temps modernes, fixent constamment la ligne d'horizon et arpentent l'espace, abandonnant au hasard l'espoir d'une rencontre.

L'exilé, constamment rétroverti sur son passé, est au contraire obsédé par l'Histoire et le sort qu'elle a réservé aux siens. D'où, dans les romans historiques marqués du fameux pacte de vraisemblance, des manies de collectionneur, l'attachement aux généalogies, à la recherche des origines ou d'un rôle historique joué par les siens, son appropriation, souvent mythifiante, de grands noms de l'Histoire universelle ou locale ${ }^{7}$, etc.

\section{Ecriture de l'errance vs écriture de l'exil}

L'exil, tout autant l'errance, peuvent constituer une riche source de création pour tout artiste. Mais de manière sensiblement différente, me semble-t-il : selon moi, l'écriture de l'errance est essentiellement tournée vers l'autre et l'ailleurs, elle est donc d'ordre centripète; celle de l'exil serait centrifuge et constamment antévertie sur un univers d'avant la rupture. Celle-ci serait d'essence mémorieuse et nostalgique; celle-là conduirait à l'exotisme qui est projection plus que rétroversion. Toutes deux ont alimenté des mythes littéraires assez significatifs des différences, que j'illustrerai ici par deux exemples :

\subsection{Du mythe de Rimbaud/Gauguin, ou mythe de l'errant}

31 Dans son "Éloge de l'errance et de la désorientation ", le géographe Luc Gwiazdzinski signale tout le profit qu'un artiste peut tirer de cette situation :

A la fois clé de lecture, posture et piste d'innovation et de créativité, l'errance est une figure stimulante dans un monde incertain. Hors là, hors les murs, hors sol, hors normes, l'errance « nous invite à être ", à habiter, à exister (...).

Voyage initiatique à la découverte de soi-même et des autres, l'errance est une épreuve qui transforme. (...)

Errer, c'est pouvoir créer de nouveaux liens et assemblages, fabriquer des sentiers, des réseaux et des imaginaires au hasard des rencontres, loin des routines du quotidien. (Gwiazdzinski, 2012 : 53)

Pour l'exemple, considérons la part qu'apportent le refus de «l'européenne vie » et la fuite vers l'inconnu - Polynésie pour l'un, Corne de l'Afrique pour l'autre - au mythe qui 
s'est progressivement constitué autour du peintre Gauguin ou du poète Rimbaud. On dispose de peu de documents sur les conditions des errances de Rimbaud en Érythrée et dans l'Arabie Heureuse, mais de Gauguin, on dispose de Noa Noa, qui affirme bien ce partipris de l'ailleurs et la force créative qu'il comporte :

Tous ces malheurs successifs, la difficulté de gagner régulièrement ma vie malgré ma réputation, mon goût pour l'exotique aidant m'ont fait prendre une détermination irrévocable. En décembre je rentrerai à Paris et je travaillerai chaque jour à vendre tout ce que je possède. Une fois le capital en poche, je repars pour l'Océanie. (...) Rien ne m'empêchera de partir et ce sera pour toujours. Quelle bête existence que l'européenne vie. (Gauguin, 1894)

Ne sous-estimons pas les fécondes ambiguïtés et contradictions de tels itinéraires ${ }^{8}$; elles sont aussi celles de l'exotisme - genre fort déprécié aujourd'hui et qui mériterait d'être réévalué au plan artistique et littéraire'. Mais je n'ai pas l'intention d'entrer dans de tels débats aujourd'hui, simplement dessiner les contours de ces mythes de l'errance, de l'artiste nomade...

\section{2. ... au mythe du Juif errant}

Pour mieux en arriver à ce mythe du Juif errant dont il sera question dans certaines communications, et qui est à mon avis mal nommé puisqu'il correspond davantage, selon ce que je viens de dire, à un mythe de l'exil infini qu'à un mythe de l'errance.

D'abord parce qu'il est né d'une condamnation, en aucune façon d'un choix individuel ou collectif. Incontestablement, le Juif errant est un perpétuel exclu plus qu'un nomade, c'est un proscrit, un banni - même si son bannissement ne s'exprime pas à l'origine dans le contexte des nations. Pardonnez-moi si je ne fais là que noter; je ne vais certes pas revenir à la genèse du mythe et à l'Evangile de Jean. D'autres dans ces pages en parleront et sans doute mieux que moi.

Mais surtout parce qu'au fil des siècles et par l'apport de l'Histoire occidentale moderne (expulsion des Juifs d'Espagne, conversions collectives forcées, introduction de thèmes nouveaux : notions de nation et de race, théories antisémites Shoah, laissés-pour-compte de l'Histoire coloniale, etc.), la légende du Juif errant va évoluer dans un sens plus politique que spirituel ${ }^{10}$. Et le Juif devenir l'éternel proscrit, l'exilé perpétuel de toutes les nations - image que les écrivains et artistes juifs (Chagall, Cohen, Wiesel,..) reprennent eux-mêmes volontiers. On connaît la formule en forme de chiasme qu'Albert Memmi attribue dans son roman Le Scorpion à son double, l'écrivain-fantôme Emile : "Ce pays hors duquel n'importe où je serai en exil... Ce pays dans lequel je n'ai jamais cessé d'être en exil. »

Même si elle peut paraître paradoxale pour un intellectuel qui se revendique à la fois Tunisien d'origine, Français de culture et de langue, de surcroît sioniste donc attaché à la nation israélienne, une telle formule illustre parfaitement la condition de ces minorités ethniques ou religieuses mises par l'Histoire moderne et contemporaine au ban des nations.

\section{Exil et dits de la mémoire}

J'en arrive à présent au quatrième et dernier point : exil et écriture de la mémoire. En fait, celui qui concerne et intéresse le plus le littéraire que je suis. Si jusqu’à présent, je n'ai 
guère approché toutes ces notions que d'un biais sociologique, je n'ai jamais eu l'intention dans mes recherches antérieures de dresser une histoire sociale ${ }^{11}$ de la mémoire maghrébine qui rapprocherait, et en même temps permettrait de distinguer, la nostalgérie pied-noir de la nostalgie des Juifs du Maghreb. Ce qui m'intéresse, ce ne sont pas les événements précis dont se souvient l'exilé, les passages obligés de telle ou telle nostalgie individuelle ou communautaire, mais comment, selon quels processus, différents et complexes, s'élabore, se découvre et se dit/s'écrit une mémoire - a fortiori chez des individus qui n'ont pas personnellement vécus ces événements - ce qui est le cas de bien des écrivains entrés en écriture ces vingt dernières années.

\subsection{Roman de l'exil et mémoire}

39 Alors que l'errant rejette toute mémoire d'un pays qu'il a fui, celle-ci accompagne l'exilé, nostalgique des origines et du pays perdu, au point que, selon Edward Saïd, « presque par définition, exil et mémoire sont des notions conjointes " (Saïd, 2008: 4ème de couv). Je vais donc m'arrêter de manière privilégiée sur ce rapport et surtout sur l'écriture de la mémoire, que j'analyse depuis une trentaine d'années sur ce corpus précis qu'est la littérature judéo-méditerranéenne.

\subsubsection{Deux exemples}

40 J'ai, il y a déjà plus de trente ans, entamé un travail sur deux romans d'un même écrivain : Albert Memmi : Le Scorpion (1969) et de Le Désert (1977), afin de montrer les enjeux très différents de la mémoire chez Jubaïr Ouali El Mammi, héros nomade du Désert, et chez des exilés comme Emile et Marcel, les deux frères narrateurs du Scorpion.

Le Scorpion apparait, typiquement, comme le roman de l'exil et de la réinvention du pays perdu par deux frères qui en ont été éloignés à l'âge adulte : l'un, Emile, est écrivain et disparaît brutalement ; l'autre, Marcel, est alors chargé de ranger ses papiers. Parmi ces monceaux d'archives en fouillis, «comment distinguer quoi que ce soit? qu'est-ce qui appartient au roman, au Journal et au reste? » En bref, où est la réalité, saisie au jour le jour par le journal intime que tenait l'écrivain, et qu'est-ce qui relève de l'imagination romanesque? Confronté à un réel insaisissable et à la reconstruction labyrinthique de souvenirs se confrontant les uns aux autres, le lecteur est abandonné à la tentation d'interprétations contradictoires et à des querelles de mémoires que l'auteur complexifie comme à plaisir d'innovations d'écriture (écriture colorée, censée faciliter la lecture, mais que j'ai pour ma part tendance à considérer comme participant de ce jeu avec un lecteur ainsi soumis à " un effort complémentaire d'imagination $»^{12}$ ) ou d'innovations narratives (polyphonie; insertion d'images et de photographies, selon un procédé particulier non d'illustration du texte mais de participation de l'image à la narration).

Le Désert est un roman historique (ou plutôt pseudo-historique) où la mémoire joue un rôle limité. Il se situe au Maghreb et dans les royaumes maures d'Andalousie et de Castille, dans un XIv ${ }^{\text {ème }}$ siècle de fantaisie. Après la destruction du Royaume-du-Dedans où il est né, le héros Jubaïr Ouali el-Mammi, qui se considère comme " un éternel étranger ", part "faire l'épreuve des hommes ", afin de s'accomplir en tant qu'homme et sans s'attacher ni à son identité car "personne n'est jamais sûr de sa naissance ", ni à son passé, car «l'enfance de l'homme ne lui appartient guère ». Son existence se transforme alors en une longue errance à travers le monde arabe, au gré des hasards et des accidents 
de l'Histoire. Si bien que lorsqu'au terme de son itinéraire il rencontre le Conquérant mongol Tamerlan, qui lui demande s'il doit faire de Balkh sa capitale, ce sont des conseils de nomade que Jubaïr lui donne: "Quiconque s'installe meurt, quel que soit le temps de l'agonie. Seigneur, n'abandonnez jamais vos tentes et vos chevaux. "

J'ai longuement étudié ailleurs ${ }^{13}$ de tels phénomènes d'appropriation sur le mode du canular, qui conduisent par exemple à présenter Jubaïr el-Mammi sous les traits et selon l'existence d'Ibn Khaldoun, héros reconnu de la civilisation arabo-berbère ; ou les mythes mélioratifs faisant de La Kahèna, qui défendit ce territoire contre les avancées arabes, une des ancêtres des Juifs du Maghreb. J'ai également évoqué les associations d'idées, les manies de collectionneurs des écrivains judéo-maghrébins et toutes les réminiscences des sens, notamment ce que j'ai appelé «la mémoire de la langue » (souvenirs olfactifs ou gustatifs, recettes de cuisines, etc.), soigneusement transmise et entretenue (Dugas, 2004 : 81-91).

\subsubsection{Une iconophilie qui s'affirme}

Dès mes tout premiers travaux, j'avais attiré l'attention sur cette spécificité de la littérature judéo-maghrébine. L'exemple du Scorpion cité plus haut met parfaitement en évidence les apports narratologiques du procédé d'insertion d'images et de photographies à l'intérieur d'un récit : la photographie est-elle garante de la vérité, autobiographique ou historique ? Par ailleurs, comment, selon quelles modalités, la photographie peut-elle prendre en charge la mémoire individuelle ou collective?

Je suis revenu plus récemment sur la question afin de montrer combien s'affirme dans l'actuelle génération d'écrivains judéo-maghrébins (Michel Valensi, Georges Chelly, Hubert Haddad, Colette Fellous,...) cette pratique d'insertion de documents, le plus souvent photographiques. Aux valeurs traditionnelles d'attestation ou de témoignage d'un vécu, s'ajoute aujourd'hui l'intérêt narratologique que revêt cette pratique, que l'on retrouve, certes, chez certains romanciers européens de l'extrême contemporain (Annie Ernaux, Jean-Philippe Toussaint,...) mais pratiquement jamais chez les écrivains maghrébins non juifs.

Colette Fellous, qui a créé aux éditions du Mercure de France la collection "Traits et Portraits" associant récits graphique et iconographique, explicite cette pratique. Pour elle, il s'agissait d'abord de constituer un environnement, " un décor ", en même temps inspirateur (en ramenant l'image d'un passé anéanti) et rassurant (en isolant d'un futur sur lequel on ne cesse de s'interroger), à l'acte d'écrire. Mais très vite elle comprend que :

tous ces documents n'étaient pas extra-littéraires, qu'ils devaient faire partie $d u$ texte, qu'ils n'étaient plus un décor mais une partie du texte lui-même. Ils n'étaient ni illustratifs, ni complémentaires du récit. Ils avaient leur propre voix, ils étaient le récit.

Et récit après récit, la possibilité d'associer texte et image agit sur l'écriture de manière étonnamment libératrice :

J'avais tous les droits, ma mémoire devenait très souple. Je jonglais avec une mémoire plus ancienne et grâce à la présence de ces photos et de ces images, je croyais me promener dans un film. Toute une partie cinématographique entrait en jeu, je pouvais raconter pour tous, au-delà de ma propre histoire, j'avais mis une distance entre mon corps et la grande Histoire grâce à cette présence de l'iconographie. (Ferrato Combes, 2009 : 57-66) 


\section{2. Écriture du post-exil}

48 Je souhaiterais pour finir me pencher avec un peu plus d'insistance sur cette littérature judéo-maghrébine des trente dernières années ${ }^{14}$, toujours préoccupée, il me semble, mais de manière fort différente, par cette écriture mémorieuse d'un passé qu'elle n'a pas directement vécu et sur lequel il ne reste pratiquement plus aucun « lieu de mémoire ».

$\mathrm{Au}$ moment où, dans le bouleversement de la fin des ères coloniales, disparait le monde juif méditerranéen, traumatisme à la fois historique et collectif, cette génération d'écrivains qu'avec Alexis Nouss nous appellerons « du post-exil » arrive en France où $\mathrm{y}$ naît parmi ceux que l'on a pris l'habitude de désigner comme la deuxième génération de l'immigration :

Contrairement à la personne exilée qui se caractérise par la recherche du homeland, la personne post-exilée ne se réfère pas à un pays d'origine comme à son lieu d'appartenance. L'expérience du post-exil la place plutôt dans une relation complexe avec des mémoires et des racines plurielles. (Nouss, 2002 : 23-24)

\subsubsection{Post-mémoire}

50 L'un des mérites de ce colloque aura été d'attirer mon attention, à travers les titres de deux ou trois communications, sur un concept qui avait échappé à ma vigilance et qui pourrait s'avérer fort opératoire : celui de post-mémoire, créé par Marianne Hirsch, à qui j'emprunte cette définition :

Le terme de postmémoire décrit la relation que la génération d'après entretient avec le trauma, collectif et personnel, vécu par ceux qui l'ont précédée. Il concerne ainsi des expériences dont cette génération d'après ne se souvient que par le biais, d'histoires, d'images et de comportements parmi lesquels elle a grandi. Mais ces expériences lui ont été transmises de façon si profonde et affective qu'elles semblent constituer sa propre mémoire. Le rapport de la postmémoire avec le passé est en vérité assuré par la médiation, non pas de souvenirs, mais de projections, de créations et d'investissements imaginatifs (Hisch, 2014 : n.p.).

51 C'est à ce prisme qu'on doit considérer l'écriture d'auteurs judéo-méditerranéens de la deuxième génération de l'exil tels que Colette Fellous (née à Tunis en 1950, arrivée en France à l'âge de 17 ans), Hubert Haddad (idem, arrivé en France à 3 ans), Gil Ben Aych (né à Tlemcen en 1948, arrivé en France avant ses 10 ans) ou Marco Koskas (né à Tunis en 1951, émigré en France en 1961, puis en Israël en 2011). Pour ceux-là, les choses se jouent de manière plus compliquée que pour leurs prédécesseurs chez qui (presque) tout s'expliquait par un déchirement entre pays perdu et nouveau lieu de résidence, avec la plongée dans les souvenirs et la quête des racines certes, mais aussi avec un présent pesant, un sentiment d'étrangeté ici et là : au drame collectif de la rupture avec le pays natal sont venus s'ajouter de nouveaux drames, à la fois plus intimes et plus terribles, mais considérés comme autant de dégâts collatéraux de la rupture initiale : la lente agonie des parents après l'exil, le suicide ou la perdition d'un frère, la dérive extrémiste d'un fils... Lisez donc pour vous en convaincre Un amour de frère (2012) de Colette Fellous, Mon cour de père (2012) de Marco Koskas, ou encore plus récemment, Les Coïncidences exagérées (2016) de Hubert Haddad. 


\subsubsection{L'Histoire à reconsidérer} Israël : refuge ultime dans un itinéraire permettant à l'exilé juif, même non d'échapper par le seul fait qu'une patrie existe au tragique de l'histoire du Juif errant (ce qui est la thèse d'Albert Memmi dans La Libération du Juif, 1966); ou pays colonialiste lui-même? Répondre à cette grave interrogation était relativement aisé pour la génération des Amran El-Maleh ou Jacob Cohen, bien intégrés dans le Maghreb indépendant et farouchement anti-israéliens, comme, à l'opposé, pour Albert Bensoussan ou Albert Memmi ayant dû fuir ce Maghreb et que leur parfaite reconnaissance professionnelle et culturelle dans leur pays d'adoption autorise à affirmer clairement leur sionisme. Ça l'est forcément moins - quelle que soit la réponse donnée et en dépit de l'intégration tout aussi problématique des Juifs maghrébins dans le nouvel état (lisez Bouganim Ami : Le cri de L'arbre, Stavit, 1983, rééd. 1996) - pour la génération post-exilique qui a rompu avec la réalité des pays arabes et qui doit vivre dans son pays d'adoption avec la montée de l'antisémitisme et du terrorisme islamique - voir l'itinéraire existentiel d'un Marco Koskas ou d'un Norbert Belange ; lire Palestine (2008) de Hubert Haddad ou les derniers romans de Chouchana Boukhobza.

\section{Conclusion}

Il y a 30 ans, j'évoquais dans ma thèse une probable «mort par dissolution » de la littérature juive en Méditerranée. Je rappelais que l'on voyait se développer «une écriture [juive] de la deuxième génération de l'immigration ", que nous nommons aujourd'hui du post-exil, «dont l'espace fictionnel est désormais largement occidental et dans laquelle la spécificité maghrébine se fait plus incidente, plus implicite et plus incertaine » (Dugas, 1991 : 63). Gilles Zenou (1957-1989), romancier judéo-marocain mort très jeune, fait dire à l'un de ses personnages que la mémoire ne produit qu'illusion «à la poursuite de l'ombre des choses plutôt que des choses elles-mêmes ", les mots euxmêmes n'offrant dès lors plus «qu'un sursis illusoire et nécessaire »(Zenou, 1989: 124). Avec la mémoire maghrébine de cette deuxième génération (que nous nommons postmémoire), nous sommes encore dans ce sursis - sans doute illusoire, car l'Histoire ne peut être réécrite...

Illusoire... mais aussi nécessaire, parce qu'il reste bien des choses à conjurer par l'écriture. Et c'est sans doute pour cela que cette littérature survit encore. Parmi les thèmes privilégiés par cette génération, je relevais les difficultés rencontrées lors de l'intégration : les ouvrages déjà cités ici, mais alors non parus pour la plupart, de Marco Koskas, de Colette Fellous de Hubert Haddad, d'Albert Memmi, de Gil Ben Aych ou de Bouganim Ami en font preuve - qui montrent sur des modes très différents des personnages incapables de s'adapter à la vie occidentale à laquelle ils aspiraient pourtant si fort. 


\section{BIBLIOGRAPHIE}

ATTALI, Jacques (2003). L'Homme nomade. Paris : Fayard.

BAUMGARTEN, Birgit, MATHIS, Ursula (dir.) (2012). Passage et ancrages en France. Dictionnaire des

écritures migrantes de langue française (1981-2011). Paris : Éd. Champion.

BISSONDATH, Neil (1994). Selling illusions : the cult of multiculturalism in Canada. Toronto : Penguin books.

BoURAoui, Hèdi (1994). Bangkok blues. Ottawa : éd. Du Vermillon.

BOURQUE, Dominique, Hogikyan, Nellie (dir.) (2010). «Introduction ", in Femmes et exils. Formes et

figures. Québec: Presses de l'université Laval.

DOSSE, François (1998). «Entre mémoire et Histoire. Une histoire sociale de la mémoire ", Raison présente, septembre 1998, pp. 5-24.

DUGAS, Guy (1991). Entre Djéha et Cagayous. La littérature judéo-maghrébine d'expression française.

Paris : L'Harmattan.

DUGAS, Guy (1995). «Vie et mort d'une littérature de l'immigration : la littérature italo-maltaise en Tunisie ", in Charles Bonn (dir.). Littérature des immigrations. vol 1 : Un espace littéraire émergent . Paris : L'Harmattan, pp. 89-101.

DUGAS, Guy (2004). « Mémoire de la langue, langue de mémoire », in Colette Zytnicki et Chantal Bordes-Benayoun (dir.). Sud-Nord. Cultures coloniales en France (XIX ${ }^{\text {ème}}$-XX siècles). Toulouse : Privat, pp. 81-91.

ETIEMBLE, René (1967, nouv. éd). Le Mythe Rimbaud, L'année du Centenaire. Paris : Gallimard.

FERRATO Combes, Brigitte (2009). « Entretien avec Colette Fellous au sujet de la collection Traits et Portraits ", in Recherches et Travaux. L'Autoportrait fragmentaire, $n^{\circ} 75,2009$, pp. 57-66.

GAUGUIN, Paul (1894). Lettre à Molard, septembre 1894.

GIDE, André (1898). «À propos des Déracinés de Maurice Barrès ", L’Ermitage, juin 1898 (repris dans Prétextes, Mercure de France, 1903).

GWIAZDZINSKI, Luc (2012). « Un possible manifeste. Éloge de l'errance et de la désorientation ", Erre, Variations labyrinthiques, catalogue de l'exposition de Metz, Centre Pompidou, p. 53.

HAGÈGE, Claude (1992). Le Souffle de la langue. Voies et destins des parlers d'Europe. Paris : Odile Jacob. HIRSCH, Marianne (2014). « Postmémoire ", Témoigner. Entre histoire et mémoire [En ligne], 118 | 2014, mis en ligne le 01 octobre 2015, consulté le 14 avril 2017,<URL : http :// temoigner.revues.org/1274> HUSTON, Nancy (1997). « Français dans le texte ", Télérama, n² 2454, 22 janvier 1997, p. 43. MAFFESOLI, Michel (1997). Du nomadisme: Vagabondages initiatiques. Paris : Le Livre de poche. MAMMERI, Mouloud (1982). La Traversée. Paris : Plon.

MASSENZIO, Marcello (2010). Le Juif errant ou l'art de survivre. Paris : éd. Du Cerf. 
MEMMI, Albert (1969). Le Scorpion. Paris : Gallimard.

MEMmi, Albert (2003). Le Nomade immobile. Paris : Arléa.

MOURALIS, Bernard (2011) [1ère éd. 1975]. Les Contre-littératures. Paris : Hermann.

Nouss, Alexis (2002). «Expérience et écriture du post-exil », in Pierre Ouellet (dir.) Le soi et

l'autre : l'énonciation de l'identité dans les contextes interculturels. Laval (Can.) : Presses de

l’Université.

ROUART, Marie-France (1988). Le Mythe du Juif errant dans l'Europe du XIX ème siècle. Paris : Corti.

SAÏD, Edward (2008). Réflexions sur l'exil et autres essais. Arles : Actes sud.

SEBBAR, Leïla (2003). Je ne parle pas la langue de mon père. Paris : Julliard.

STASZAK, Jean-François (2004). «L'exote, l'oviri, l'exilé : les singulières identités géographiques de Paul Gauguin // The exote, the oviri and the exiled : Gauguin's singular geographical identitites ", Annales de Géographie, t. 113, n638-639, pp. 363-384.

\section{NOTES}

1. Dans cette contribution liminaire au volume collectif Convergences et divergences dans les littératures francophones (Paris, l'Harmattan, 1992), pp. 15-21, j'avais reconsidéré les notions de francophonie, d'acculturation, de littératures nationales ou minoritaires, etc.

2. J'ai étudié ce dernier cas dans Dugas, 1995 : 89-101.

3. «L'exilé-e, typiquement, est nostalgique ; il ou elle aspire au retour au pays natal, au homeland. Il ou elle se sent déchiré-e et survit entre le pays quitté et le nouveau lieu de résidence.» (Bourque, Hogikyan, 2010 : 17).

4. Neil Bissondath (1994 : 26), Canadien d'origine trinidadienne, prétend de son côté : «My roots travel with me in my pocket $»$.

5. Voir à ce sujet Claude Hagège (1992).

6. Voir aussi le court mais riche entretien de l'auteur avec Taïna Tervonen : <URL : http:// africultures.com/je-ne-parle-pas-la-langue-de-mon-pere-2817/> [consulté le 31/10/2016]

7. Je pense par exemple à la déformation onomastique (Cahéna au lieu de Kahèna) et, par voie de conséquence, de condition, que certains écrivains judéo-maghrébins font subir au personnage mythique de Diya, figure mythique de la résistance berbère à la conquête arabe au VIIème siècle - ce qui subvertit l'Histoire maghrébine et constitue, par l'imaginaire, une appropriation de cette grande figure de l'Ifriquiya.

8. Sur Rimbaud, on pourra consulter René Etiemble (1967). Sur Gauguin, Staszak (2004 : 363-384).

9. Bernard Mouralis voit dans la démarche de l'exote un "acte de courage d'abord» correspondant à un choix éthique qui se caractérise par «une récusation du monde qui jusqu'alors était le sien », afin de partir à la rencontre d'un autre. (Mouralis, 2011 : 101)

10. Voir à ce sujet Rouart (1988) et Massenzio (2010).

11. L'expression est de François Dosse (1998 : 5-24).

12. Note de l'auteur, Le Scorpion, p. 295. Ce n'est pas ici le lieu de discuter la théorie d'Albert Memmi sur l'écriture colorée. Je renvoie donc à son petit essai : L'Ecriture colorée ou je vous aime en rouge. Paris, Périples, 1986 et à mon article «Quelques compléments à propos de l'écriture colorée chez Albert Memmi » dans Sephardic Horizons, revue en ligne: http:// www.sephardichorizons.org/Volume5/Issue3-4/Dugas.html

13. Voir ma thèse : Dugas (1991). Et sur la production la plus récente, mon article : «Fantaisie, littérature mémorieuse, iconophilie... Retours sur quelques concepts discutables », in Expressions 
maghrébines, vol. 13, $\mathrm{n}^{\circ}$ 2, hiver 2014, « Nouvelles expressions judéo-maghrébines », sous la direction de Sonia Zlitni Fitoussi et Guy Dugas.

14. J'ai récemment prolongé ma Bibliographie méthodique et critique de la littérature judéomaghrébine d'expression française (1896-1982), Paris, l'Harmattan, 1991 d'une bibliographie de «Trente ans de littérature judéo-maghrébine (1982-2013) », dans Expressions maghrébines, op.cit. 15. J'ai traité cette question de manière détaillée dans «Palestine et Israël. Sionisme et alyah dans la littérature judéo-maghrébine d'expression française ». Publ. en ligne : https:// www.academia.edu/7415347/Sionisme_et_alyah_dans_la_litterature_judeo-maghrebine_ dexpression_francaise

\section{RÉSUMÉS}

Si l'ensemble qui suit peut être lu comme une somme de variations autour des notions d'errance, d'exil et de mémoire, cette contribution, qui définit ces divers concepts et en souligne les prolongements possibles dans le vécu comme dans les pratiques artistiques, en constitue les nécessaires prolégomènes. Venant de la part d'un universitaire qui a consacré l'essentiel de sa recherche aux expressions minoritaires en contexte de domination coloniale, cette introduction prend aussi la forme d'un bilan et ouvre quelques pistes de recherche, à partir de notions nouvellement apparues telles que celles de post-exil et post-mémoire.

If the following opus can be read as a survey of changes around the notions of wandering, exile and memory, this contribution, which defines these varied concepts and underlines their possible ramifications both in life and in artistic practice, provides the prefatory remarks which they require. Coming from an academic who has dedicated the greater part of his research to minority cultures in colonial context, these introductory remarks may also be taken as a summary which opens several avenues of research, beginning with recently-expressed concepts such as these of postexile and postmemory.

\section{INDEX}

Mots-clés : errance, nomadisme, exil/post-exil, mémoire/post-mémoire

Keywords : wandering, nomadism, exile/postexile, memory/postmemory

\section{AUTEUR}

GUY DUGAS

Professeur émérite, Université Montpellier 3

dugas.montp3[at] gmail.com 